\title{
Analysis of the Breakdown and Electrical Arc in the Process of Switching on
}

\author{
Łukasz Kolimas
}

\begin{abstract}
Switching on current in an electric circuit occurs hence not due to securing the contact of contacts, but as a result of electric break down of the isolation of the given environment. The switching-on arc glowing time depends hence on the value of the intensity of electric field in the contact area, and on the velocity of contacts closing. The paper describes the analysis of the electrical fields and problems of the arc switching time in contact switches during making of a current in current paths and test.
\end{abstract}

Index Terms - electric field strength, electric arc, electrical contacts.

\section{INTRODUCTION}

Closure of contacts in any environment which constitutes switch insulation gap, causes a breakdown of the isolation layer, and an arc appears between the approaching contacts. Switching on current in an electric circuit occurs hence not due to securing the contact of contacts, but as a result of electric break down of the isolation of the given environment. In the process of switching on the high current, especially short-circuit currents, the contacts of the electric switch heat up intensely as a result of electric arc ignition, and current flow [2-6]. Assuming that the course of short-circuit current in pole of switch is defined by $\mathrm{i}(\mathrm{t})$, the energy emited in electrical arc $\mathrm{W}_{\mathrm{a}}$ during of make-time $\mathrm{t}_{\mathrm{a}}$, can be determined from relationship:

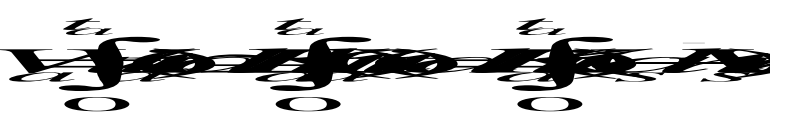

where:

$\mathrm{u}_{\mathrm{a}}$ - electrical arc voltage drop, $\mathrm{V}$,

$\mathrm{i}(\mathrm{t})$ - short-circuit current, $\mathrm{A}$,

$\mathrm{K}_{\mathrm{a}}$ - gradient of arc, $\mathrm{V} / \mathrm{m}$,

$\mathrm{n}$ - number of the contact gaps in one pole of a switch,

$\mathrm{t}_{\mathrm{a}}$ - the switching-on arc duration, $\mathrm{s}$,

$\mathrm{d}_{\mathrm{s}}-$ distance between contacts, $\mathrm{m}$,

$\bar{V}_{s}$ - average value of the velocity of contacts closing,

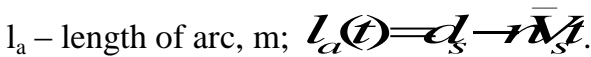

So, the higher the value of velocity $\mathrm{v}_{\mathrm{s}}$ of contact closing and electrical field strength, the shorter the turn-on time.

\section{THE ELECTRIC FIELD STRENGTH}

For the sake of determination of the distribution of electric field intensity in the contact zone, and location of the contact areas utmost exposed to puncture, the finite element method has been applied. Because of axial symmetry present in the considered contact systems, Quick Field [1] software has been used.

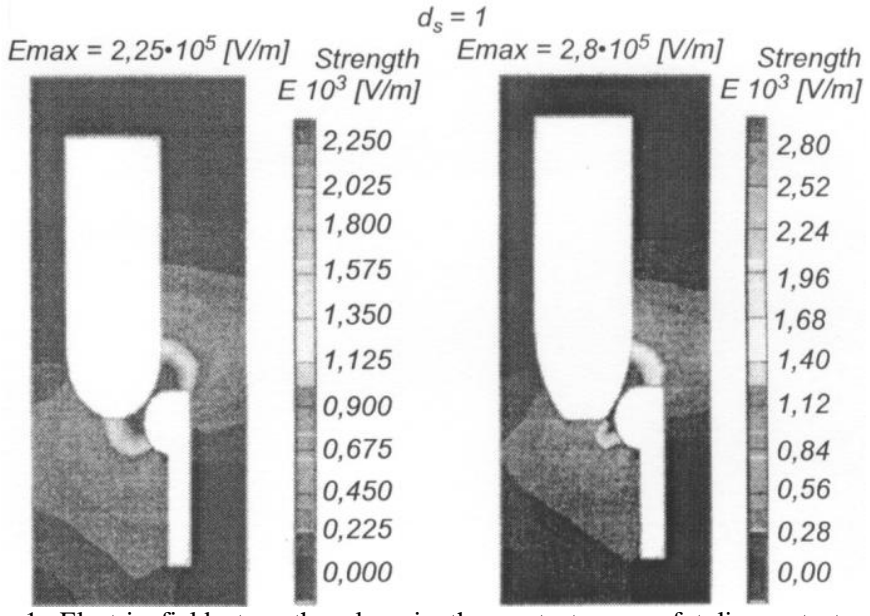

Fig. 1. Electric field strength values in the contact zone of tulip contact systems, at contact gap $\mathrm{y}=1 \mathrm{~mm}$.

The object of investigations were mostly tulip and conical contacts with various shapes of tips of movable and immovable contacts. Example values of the electric field strength values in the contact zone of tulip contact systems have been presented in fig. 1 . It has been found out that important design features influencing the distribution of electric field in the contact. It has been also determined [2] that the value of slant angle $\alpha$ of conical tulip contact has a relatively small influence on the electric field strength value in the contact gap. Some rise of the electric field value in the contact zone corresponding to the increase of angle $\alpha$ is observed only in the last phase of the closing process. The influence of the number of contact points of the tulip stationary contact upon the electric field intensity value has not been confirmed.

The breakdown of the contact gap occurs at the moment of equalising of contact gap dielectric strength $u_{p}\left(t_{s}, t\right)$ with the momentary value of the voltage $u(t)$ applied to the gap (Fig. 2). 


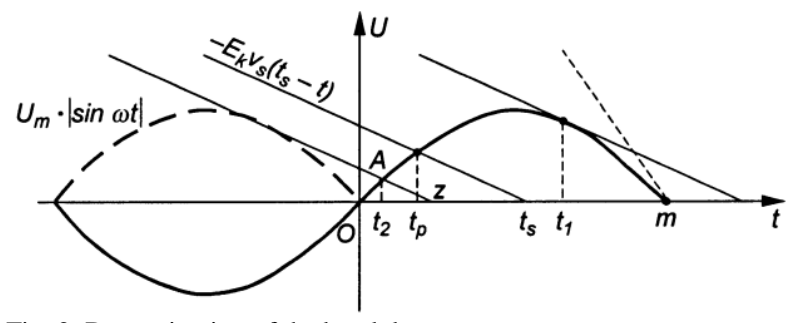

Fig. 2. Determination of the breakdown moment.

Assuming that the breakdown voltage is proportional to the distance between the contacts, and that it is not polaritydependent, it is possible to determine the time $t_{p}$ when the breakdown occurs during current switching on from the relation:

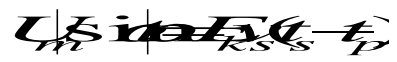

where:

$\mathrm{E}_{\mathrm{k}}-$ critical value of electric field strength,

$\mathrm{t}_{\mathrm{p}}$ - moment of break down of contact gap,

$\mathrm{t}_{\mathrm{s}}-$ moment of contact closing.

In the operation of switching on an alternating current circuit, the signal to make the electric switch usually is not correlated with the voltage phase, so breakdown may occur at various distances between contacts, and at various moments, i.e. at various voltage phase angles. Hence, the arc duration may be different for each current switching on. The arc duration $t_{a}=t_{s}-t_{p}$ depends on the velocity $v_{s}$ of contact closing, and on the electric field strength value $\mathrm{E}_{\mathrm{k}}[1]$, [2]. In flat face contact arrangements the velocity $\mathrm{v}_{\mathrm{s}}$ of the contact gap decrease is equal to the velocity $v_{y}$ of the axial motion of the moving contacts (Fig. 3).

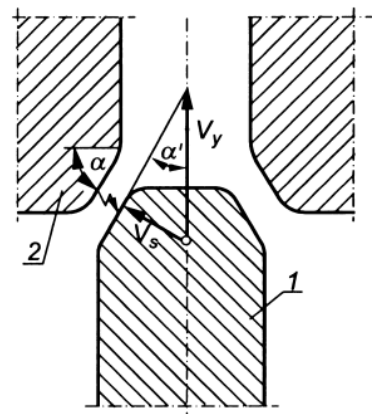

Fig. 3. Example of the conical contact tip: 1 - moving contact, 2 - stationary contact.

The arc duration depends on the value of electric withstand against contact gap breakdown and on the value of velocity $\mathrm{v}_{\mathrm{s}}$ of contact closing. In the given conditions, this time will be shorter, the closer to zero value the voltage phase is.

For the sake of switching the current on in an electric circuit at the exact moment of voltage, i.e. to avoid the breakdown of contact gap, two practical conditions must be fulfilled [2]:
1. the dynamic withstand of the contact gap during contact closing should be higher than the instantaneous value of the supply voltage;

2. the scatter of making time of electric switch $t_{c}=t_{p}+t_{a z}$ should be possibly lowest; for making switches the scatter of making time values should be considered satisfactory if:

$$
\Delta t_{c} \leq 5^{\circ} e l
$$

Switching on is hence at any voltage phase angle (fig. 2) including the phase angle corresponding to the moment of voltage passing the zero value, provided that the condition for $\mathrm{k}$, is satisfied:

$$
k=\frac{E_{k}^{v}{ }_{s}}{\omega U_{m}} \geq 1
$$

The selection of a determined phase of switching current on requires the application of an electronic system to control the process of the switches, e. g. making switch or synchronised switch.

\section{ELECTRIC FIELD ANALYSIS}

It is possible also to use the displayed model for examining preserving the arc in the presence of shunting elements field strength. In figure 4 an amendment to the schedule of straining electromagnetic fields at applying shunting elements was described.

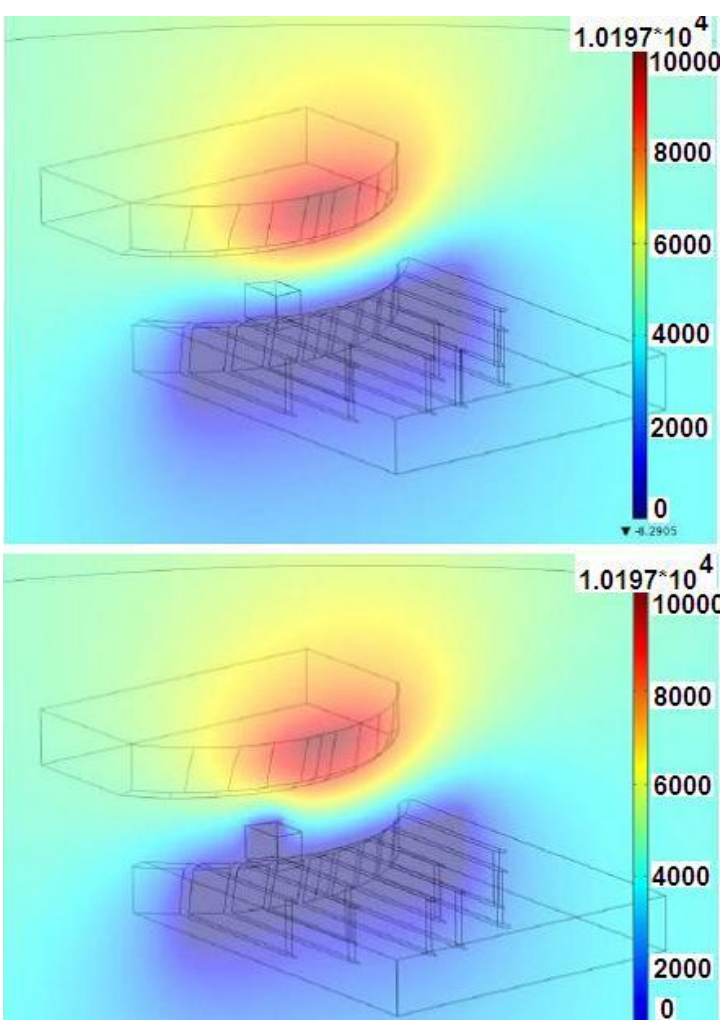

Figure 4. Amendment to the schedule of the magnetic field strength at applying the element shunting in the arrangement of the short-circuit attachment. Disintegration on the height of the head of the shunting element. 
The idea of using shunting elements and their influence on dispersing field in pin arrangements was described in literature [3]. In order to element shunting could fulfil one's role must be made of material being characterized by maximum values of the electric penetrability of relative $\square$. Group of materials about the high electric penetrability relative there are ceramics of the type PZT (Fig. 5) obtained from the sinter of powders of titanium-zirkonian of lead.

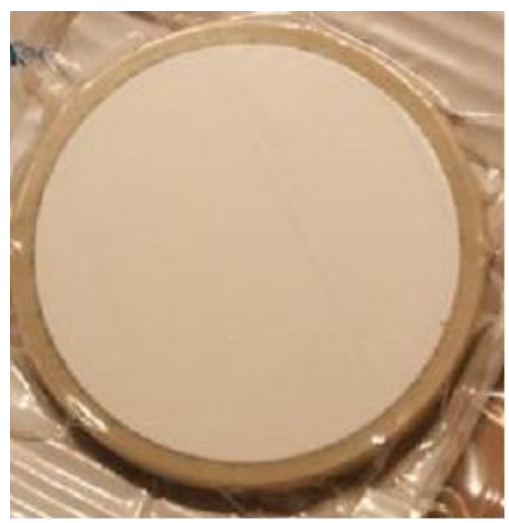

Figure 5. Element of the type PZT SONOX P5 of the Ceram Tec AG production.

PZT materials are piezoelectric materials and their properties closely depend on the constancy of the polarization. The majority of the property of elements is undergoing the gradual decline in the time. Exact rates of the decline depend on the composition of ceramic elements and the production technology. Improper applying elements by overstepping electric, mechanical or thermal restrictions can precipitate this inevitable process. Electric restrictions - surrendering to the influence the strong electric field, about the opposite polarity in relation to the field of the primitive polarization, will cause the depolarization of material called piezo. The stair of the depolarization depends from the kind of material, time of the influence, temperature and other factors, however influence of $200-500 \mathrm{~V} / \mathrm{mm}$ or bigger usually causes the depolarization. The alternating current causes the depolarization during every of half cycle, in which the polarization is against a primitive polarization of the element for field. Mechanical restrictions mechanical stresses satisfactories for disturbing the sense of direction domain can supply material for destroying tidying dipoles up. The same as the susceptibility to the electric depolarization, the resistance to stresses is mechanical diversified of different grades of materials piezo. Thermal restriction - if piezo material piezo gonna be heated to the Curie temperature, domain are becoming unordered, and material gonna be polarized. The recommended upper operating temperature of ceramics is falling out approximately half between $0 \mathrm{oC}$ and the Curie temperature. Kind of applied additives of piezo materials is deciding on their properties. Socalled soft ceramics are demonstrating high electromechanical, high coupling permanent piezoelectric, high electric relative, high penetrability permanent dielectric, high dielectric losses, low coefficient of the mechanical goodness and mean the nonlinearity. Soft ceramics will withstand greater transfers towards so-called hard ceramics, however is demonstrating the bigger hysteresis and the greater susceptibility to the depolarization. Minimum values of the Curie temperature cause applying soft ceramics in applications requiring the high-frequency and the strong electric field. Therefore, soft ceramics mainly are being used in sensor applications. Hard ceramics generally are holding properties opposite to soft ceramics, including maximum values of the Curie temperature, low permanent loading, low electromechanical link, high coefficient of the electromechanical goodness. It is also less susceptible to the polarization and the depolarization. Although hard ceramics are showing the greater stability from soft ceramics, isn't able to produce this way of great transfers. Hard ceramics are suitable for applying in applications requiring big stresses and high voltages.

\section{CONCLUSIONS}

The arc duration $t_{a}=t_{s}-t_{p}$ depends on the velocity $v_{s}$ of contact closing, and on the electric field strength value $\mathrm{E}_{\mathrm{k}}$.

Numerical analysis of electric field strength distribution in the contact gap of contact systems by the means of finite element method is useful, especially when it comes to definition of details of the contact system initiating the breakdown of insulation gap.

\section{REFERENCES}

[1].E. Kuffel, W. Zaengl, J. Kufel, High Voltage Engineering, Butterworth-Heinemann, 2000.

[2]. S.J. Kulas, "Analysis of the transient states in the closing contacts," Proceedings of SAP 2001, Switching Arc Phenomena, pp. 236240, Łódź, Poland, 2001.

[3].P.G. Slade, "Electrical Contacts for Power Interruption," Proceedings of ICEC'98, Int. Conf. On Electric Contact Phenomena, Nuremberg, Germany, pp. 239-245, 1998.

[4].X. Zhou, P. Theisen, "Investigation of arcing effects during contact blow open process," IEEE Transactions on Components and Packaging Technologies, vol.23, № 2, pp. 271-277, 2000.

[5]. Kolimas L., Kulas S.: The Physical Phenomena in Closing Contacts of Electrical Switches, XIX Symposium on Physics of Switching Arc (FSO 2011), Brno, Czech Republik 2011, Conference proceedings, ISBN 978-80-214-4293-1, pp 247-250.

[6]. Kulas S., Kolimas Ł.: Optimization of Electric Field in Contacts on Example of Making Switch, Proceedings of the $42^{\text {th }}$ International Universities Power Engineering Conference, University of Brighton, Brighton, UK 2007, pp. 491-495.

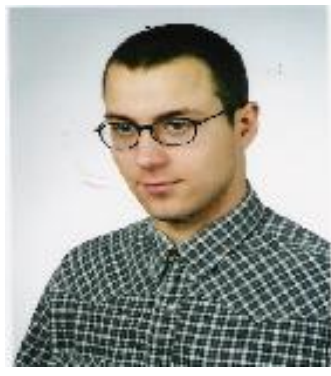

Lukasz Kolimas is a $\mathrm{PhD}$ Engineer, a Science officer in Warsaw University of Technology, Decision of Power System Apparatus, Protection and Control Personal economic activity related with professional technical counseling from 2007 to present. He was a PhD Student in Warsaw University of Technology, with a major in Electrical Engineering under the 
Electrical Apparatus Department from 2005-2006. He studied in Warsaw University of Technology (Power System and Robotics) and coordinated studios automation of drive from 2000-2005.

He practiced in PGNiG (Polish Oil and Gas Company) from Czerwiec 2002 - wrzesueń 2002. He was a Conservator of elevator from Czerwiec 2003 - wrzesień 2003 and a Counselor on OSRAM Company from 2006 - 2008 before being engaged in a Personal economic activity in 2008. 